\title{
Noninvasive cardiac output monitoring: pulse wave transit time (PWTT) versus pulse waveform analysis during elective cardiac surgery
}

Suraphong Lorsomradee, M.D., Ph.D. ., Sratwadee Lorsomradee, M.D.

Department of Anesthesiology, Chiang Mai University Hospital, Thailand.

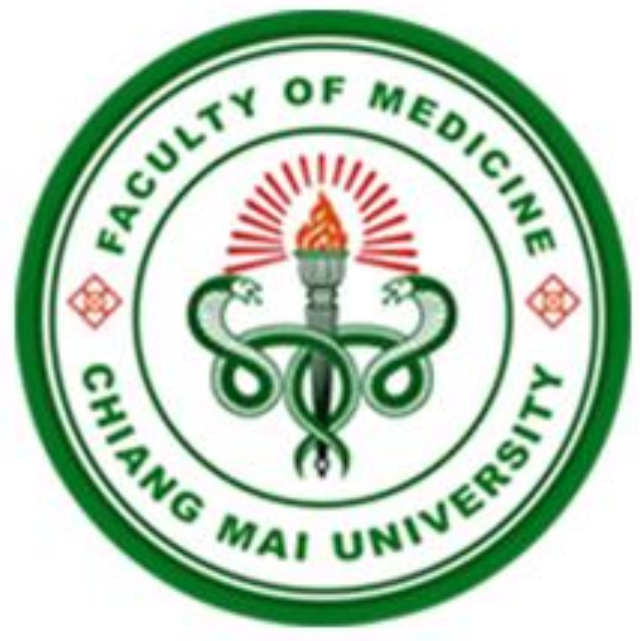

BACKGROUD: The newest noninvasive method for estimated continuous cardiac output (esCCO) measurement uses a technique based on the relationship between pulse wave transit time (PWTT) and stroke volume. The noninvasive device provides esCCO measurements using the routine electrocardiogram (ECG), pulse oximeter wave, and arterial blood pressure. This study evaluated the overall efficacy of noninvasive esCCO using PWTT compared with the arterial pressure contour analysis (APCO) during cardiac surgery.

MATERIALS AND METHODS: Twenty elective cardiac surgery patients were received routine monitoring. The radial arterial blood pressure were attached to a sensor (FloTrac, Edwards Lifesciences, Irvine,CA) has a bifurcated cable with one going to the BSM-9101 (Nihon Kohden, Tokyo, Japan) bedside monitor to display routine arterial blood pressures and esCCO, and the other going to the Vigileo monitor to analyze for the APCO and SVV. All hemodynamic data including bedside monitor, vigileo and esCCO will be automatically recorded simultaneously into the software during the entire cardiac operation.

Figure1. Cardiac output alteration during routine cardiovascular surgical interventions

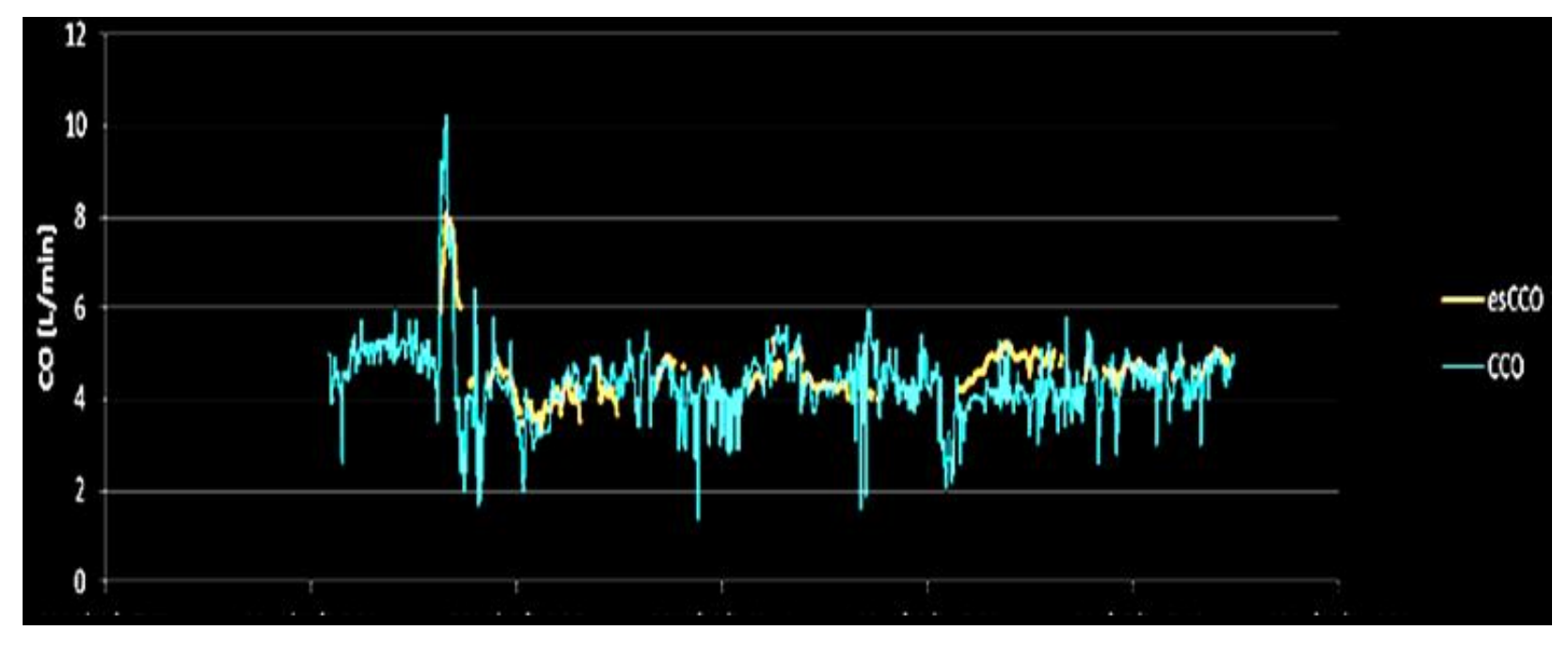

Figure2. Correlation between esCCO and APCO.

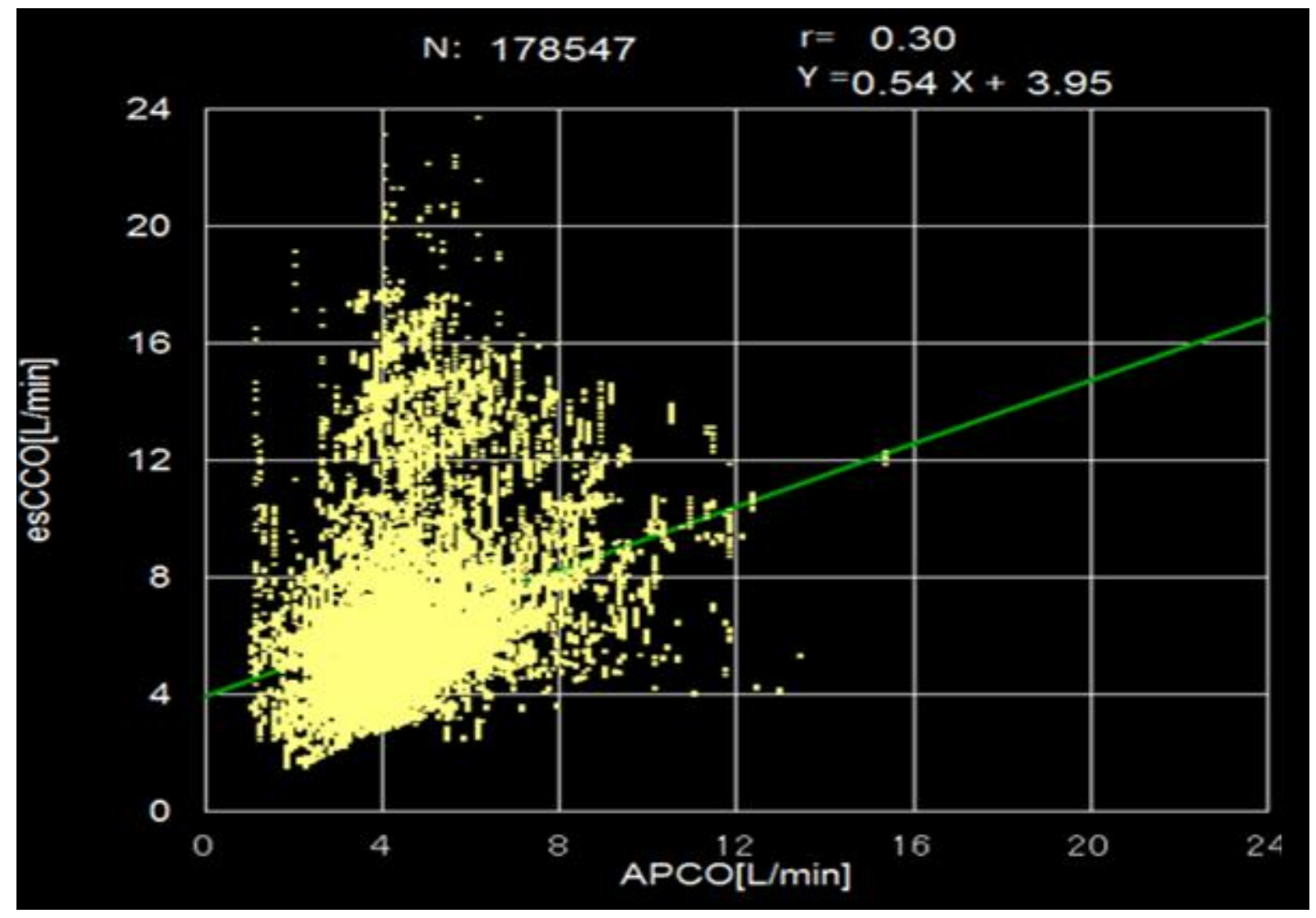

RESULTS: The esCCO data was frequently interfered with intermittent electrocauterization and cardiac surgical manipulation which resulting the ECG-oxygen saturation signal interferance. A total of 178,547 pairs of simultaneous cardiac output measurements were recorded. The esCCO showed a poor correlation with the APCO $\left(R=0.3\right.$; esCCO $=\left[0.54^{*} \mathrm{APCO}\right]$ $+3.95)$. Bland-Altman analysis showed poor overall agreement between the two methods. Bias (limits of agreement) was 1.87, precision was 2.77, and \% Error was 123. However, fluid challenge test increase APCO $34.1 \% \pm 18 \%$, whereas it increase esCCO by $13.3 \% \pm 1 \%$.

CONCLUSION: The esCCO showed a poor correlation with the APCO during perioperative hemodynamic instability of cardiac operation. However, the trend changes after fluid challenge test were in the same direction.

Figure3. Bland-Altman analysis showed overall agreement between esCCO and APCO.

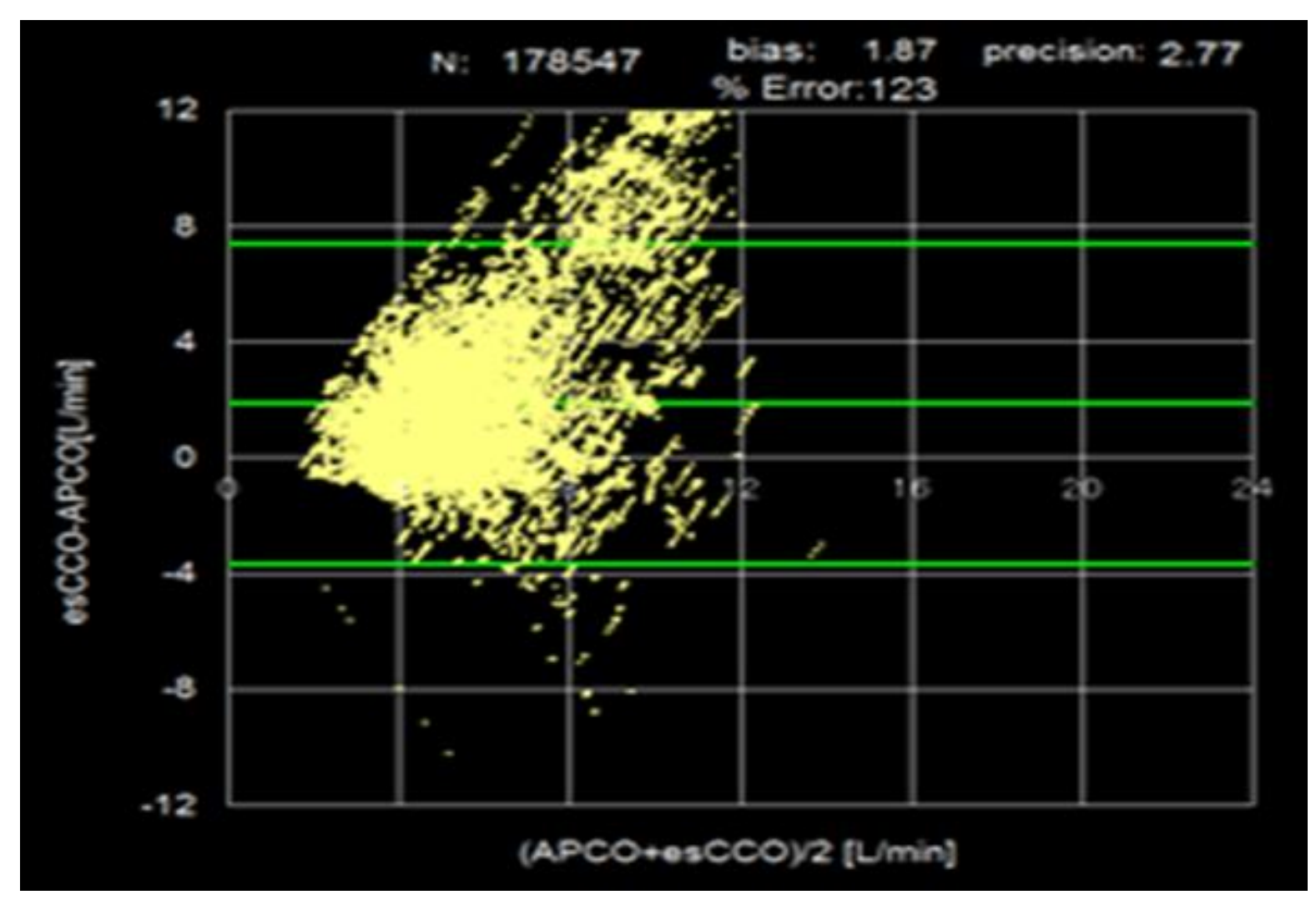

Figure4. Fluid challenge test.

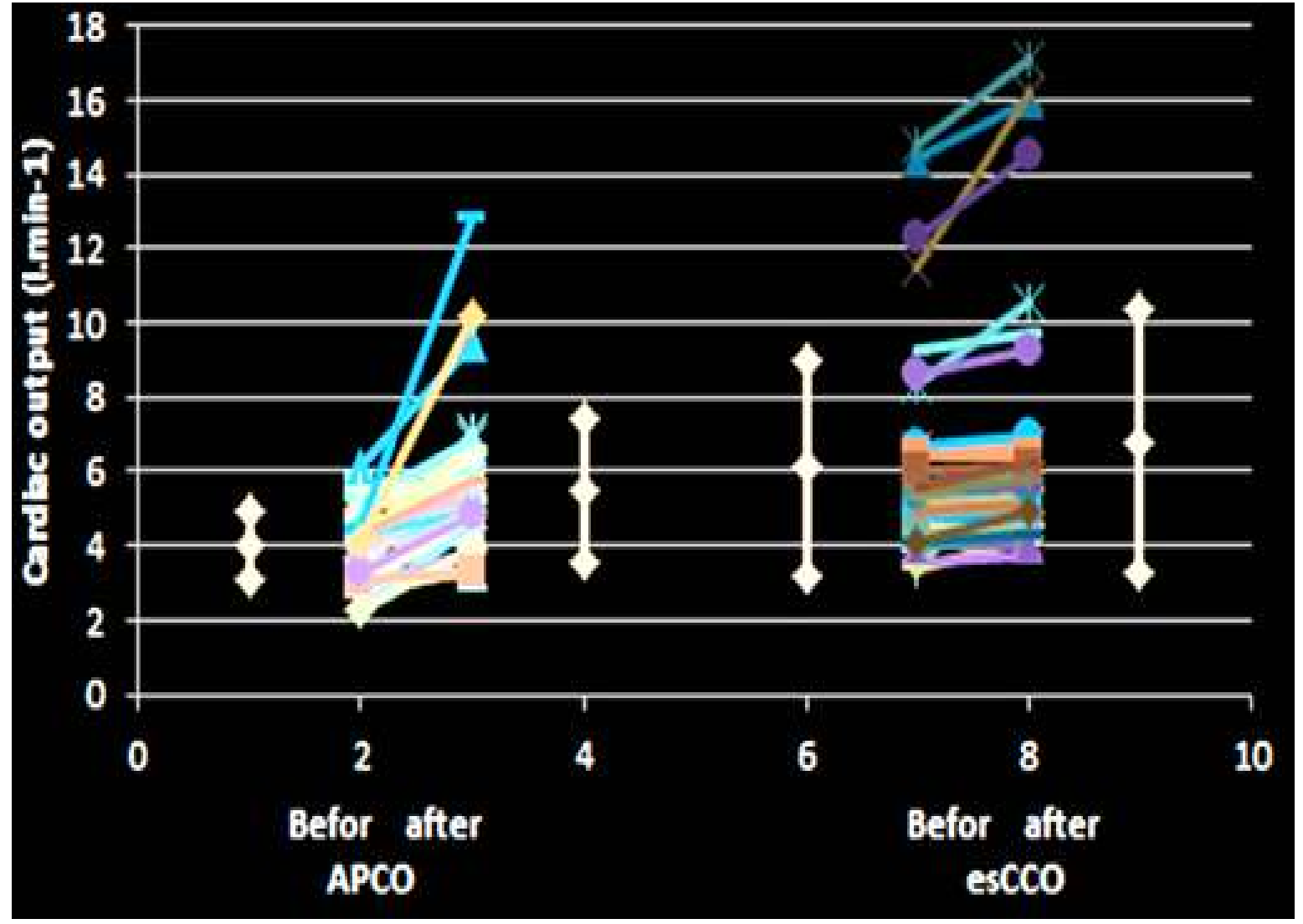

